\section{Disparate Rates of Molecular Evolution in Cospeciating Hosts and Parasites}

\author{
Mark S. Hafner, Philip D. Sudman, Francis X. Villablanca, \\ Theresa A. Spradling, James W. Demastes, Steven A. Nadler
}

DNA sequences for the gene encoding mitochondrial cytochrome oxidase $I$ in a group of rodents (pocket gophers) and their ectoparasites (chewing fice) provide evidence for cospeciation and reveal different rates of molecular evolution in the hosts and their parasites. The overall rate of nucleotide substitution (both silent and replacement changes) is approximately three times higher in lice, and the rate of synonymous substitution (based on analysis of fourfold degenerate sites) is approximately an order of magnitude greater in lice. The difference in synonymous substitution rate between lice and gophers correlates with a difference of similar magnitude in generation times.

Chewing lice of the genera Geomydoecus and Thomomydoecus are obligate ectoparasites of pocket gophers (Fig. 1). Because the entire life cycle of these lice occurs exclusively in the fur of the host, and because different host species rarely interact, each species of louse is normally restricted to a single host species (1). As a result, there is close correspondence between gopher taxonomic boundaries and louse taxonomic boundaries (2). When viewed over large geographic and temporal scales, this restricted distributional pattern of chewing lice on pocket gophers has resulted in phylogenetic histories of lice and gophers that are remarkably similar (3-5).

Although well-documented cases of host-parasite cospeciation are rare $(3,6)$, they are of interest because they permit comparative study of organisms with a long history of parallel evolution. The temporal component of parallel phylogenesis (in which lineages of hosts and their parasites speciate repeatedly at approximately the same time) permits examination of relative rates of evolution in the two groups by comparison of the amount of change each has undergone during their parallel histories. Because the life histories of hosts and their parasites are often profoundly different, studies of molecular evolution in hostparasite assemblages can help answer a broad spectrum of questions relating to the possible effects of generation time, metabolic rate, and other life history parameters on rates of mutation and evolutionary change.

We examined DNA sequence variation in 14 species of pocket gophers and their chewing lice (7) to test for cospeciation and to investigate rates of molecular evolution in this host-parasite assemblage. We sequenced and compared homologous regions of the gene encoding the mitochondrial cytochrome c oxidase subunit $\mathrm{I}(\mathrm{COI})$ in both groups (8). Of the 379 nucleotides sequenced for each taxon, 134 positions were variable in pocket gophers and 178 positions were variable in chewing lice ( $\mathrm{Ta}$ ble 1).

The cospeciation hypothesis predicts that the branching structure of the host and parasite phylogenies will be similar to a degree beyond that expected to occur by chance. This prediction can be evaluated statistically $(3,4)$. For any particular host-parasite assemblage, confidence in the test of cospeciation can be no stronger than confidence in the phylogenies under comparison. Thus, it is essential that the host and parasite phylogenies accurately estimate the evolutionary history of each group. There are many methods for estimating phylogenies from sequence data (9), each of which uses a different model of nucleotide evolution and potentially yields a phylogenetic hypothesis (a tree) that differs from that estimated with other methods (10). To consider the effects of different evolutionary models on our estimates of phylogeny, we applied multiple methods of analysis $(11,12)$ to our se-

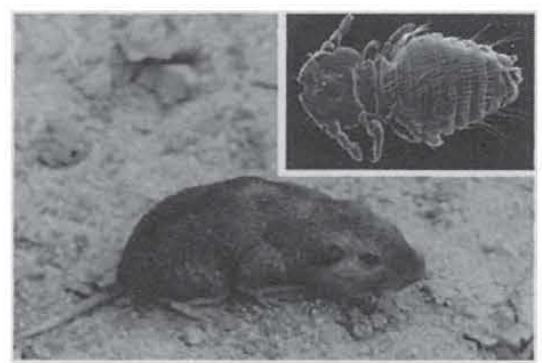

Fig. 1. Chewing lice (Geomydoecus texanus, inset) are wingless insects that are obligate ectoparasites of pocket gophers (Thomomys bottae is shown here). The entire life cycle of the chewing louse occurs in the fur of these fossorial rodents. quence data. In cases where different methods yielded different results, we retained all host and parasite trees for topological comparison in order to determine whether the inference of cospeciation is warranted and, if so, whether the inference is sensitive to the method of analysis.

All analyses of the pocket gopher sequence data (using different models of DNA sequence evolution) yielded trees that were very similar in overall branching structure. For example, phylogenetic analysis (11) of the COI sequence data for pocket gophers yielded two most-parsimonious trees of equal length (1423 steps). One of these trees (Fig. 2A) was topologically identical to the tree generated by a maximum-likelihood analysis of the same data (12). The other most-parsimonious tree showed only minor differences (13) from the tree shown in Fig. 2A. The general structure of the gopher parsimony tree (Fig. 2A) also was supported by Fitch-Margoliash (Fig. 2B) and neighborjoining (14) analyses of genetic distances (12). Differences among the trees generated by the parsimony, maximum-likelihood, Fitch-Margoliash, and neighborjoining analyses of the pocket gopher data were judged nonsignificant by a likelihood ratio test (15). Accordingly, all four trees were retained for topological comparison with the parasite trees. The basic structure of these trees and, in particular, relations within the genera Orthogeomys and Geomys, also are supported by inde-

Table 1. Observed percent of difference (mean $\pm 1 \mathrm{SD}$ ) in various elements of the COI nucleic acid sequence from pocket gophers and their ectoparasitic chewing lice.

\begin{tabular}{|c|c|c|}
\hline & \multicolumn{2}{|c|}{$\begin{array}{l}\text { Percent of sequence } \\
\text { difference }( \pm 1 \mathrm{SD})^{*}\end{array}$} \\
\hline & Gophers & Lice \\
\hline $\begin{array}{l}\text { First position } \\
\text { transitions }\end{array}$ & $1.43(0.49)$ & $2.09(0.62)$ \\
\hline $\begin{array}{l}\text { First position } \\
\text { transversions }\end{array}$ & $0.00(0.25)$ & $0.46(0.34)$ \\
\hline $\begin{array}{l}\text { Second position } \\
\text { transitions }\end{array}$ & $0.24(0.18)$ & $0.38(0.37)$ \\
\hline $\begin{array}{l}\text { Second position } \\
\text { transversions }\end{array}$ & $0.00(0.00)$ & $0.16(0.17)$ \\
\hline $\begin{array}{l}\text { Third position } \\
\text { transitions }\end{array}$ & $8.74(1.65)$ & $9.59(1.51)$ \\
\hline $\begin{array}{l}\text { Third position } \\
\text { transversions }\end{array}$ & $5.01(1.67)$ & $7.68(1.99)$ \\
\hline Total difference & $15.64(3.20)$ & $20.75(2.31)$ \\
\hline $\begin{array}{l}\text { Silent nucleotide } \\
\text { differences }\end{array}$ & $14.75(3.09)$ & $17.66(2.32)$ \\
\hline $\begin{array}{l}\text { Replacement } \\
\text { nucleotide } \\
\text { differences }\end{array}$ & $0.89(0.71)$ & $2.67(1.05)$ \\
\hline $\begin{array}{l}\text { Amino acid } \\
\text { differences }\end{array}$ & $2.40(1.83)$ & $6.85(2.62)$ \\
\hline
\end{tabular}


pendent phylogenetic studies based on morphology, allozymes, comparative immunology, karyology, and nucleotide sequence data $(3,5,16-18)$.

All analyses of the chewing louse sequence data likewise yielded trees with similar branching structures. Phylogenetic analysis of the louse COI data yielded three most-parsimonious trees of equal length (4208 steps). One of these trees (Fig. 2A) was topologically identical to the tree generated by a maximum-likelihood analysis of the same data. The two remaining parsimony trees showed only minor differences (involving one species in each case) from the tree in Fig. 2A (19). The Fitch-Margoliash analysis (Fig. 2B) and the neighbor-joining analysis (20) yielded louse trees very similar to those generated by the parsimony analysis. Differences among the trees generated by the parsimony, maximum-likelihood, Fitch-Margoliash, and neighbor-joining analyses of the louse data were judged nonsignificant by a likelihood ratio test (15). Accordingly, all five louse trees were retained for topological comparison with the host trees. The basic structure of the louse trees and, in particular, relations among lice hosted by species of Orthogeomys and Geomys, also are supported by independent phylogenetic studies of allozymes $(3,5,21)$.

The COMPONENT program (22) determined if the fit between observed parasite and host trees was significantly better than the fit between the parasite tree and trees drawn at random from the set of all possible host trees (4). For each of 20 pairwise comparisons (four host trees and five parasite trees), the observed degree of fit between the gopher and louse trees was significantly better $(P<0.01)$ than the fit between the louse tree and 10,000 randomized gopher trees (23). These results, which are robust to the method of phylogenetic inference and to the evolutionary models used, falsify the null hypothesis of chance similarity between the host and parasite trees. Although this evidence is consistent with the hypothesis of cospeciation, the concordant phylogenies might instead result from dispersal, extinction, or incomplete sampling of closely related taxa (4). However, only the cospeciation hypothesis predicts temporal congruence of host and parasite speciation events, which (given roughly time-dependent molecular change in each group) would result in a significant relation between measures of molecular differentiation in the host and parasite trees. We demonstrate below that our molecular data are consistent with this prediction. This finding, which requires no assumptions about rate similarity between hosts and parasites (3), corroborates independent evidence for cospeciation in several genera of pocket gophers (Orthogeomys, Geomys, and Thomomys) and their lice (3-5).

Given evidence for cospeciation, it is possible to test the null hypothesis that pocket gophers and chewing lice have undergone equivalent amounts of genetic differentiation during their parallel histories. It is appropriate that this test be restricted to hosts and parasites that have cospeciated, because time since divergence can be assumed to be equal only for host-parasite pairs that show cospeciation ( 9 host-parasite pairs in Fig. 2A and 10 host-parasite pairs in Fig. 2B) (24). We first compared
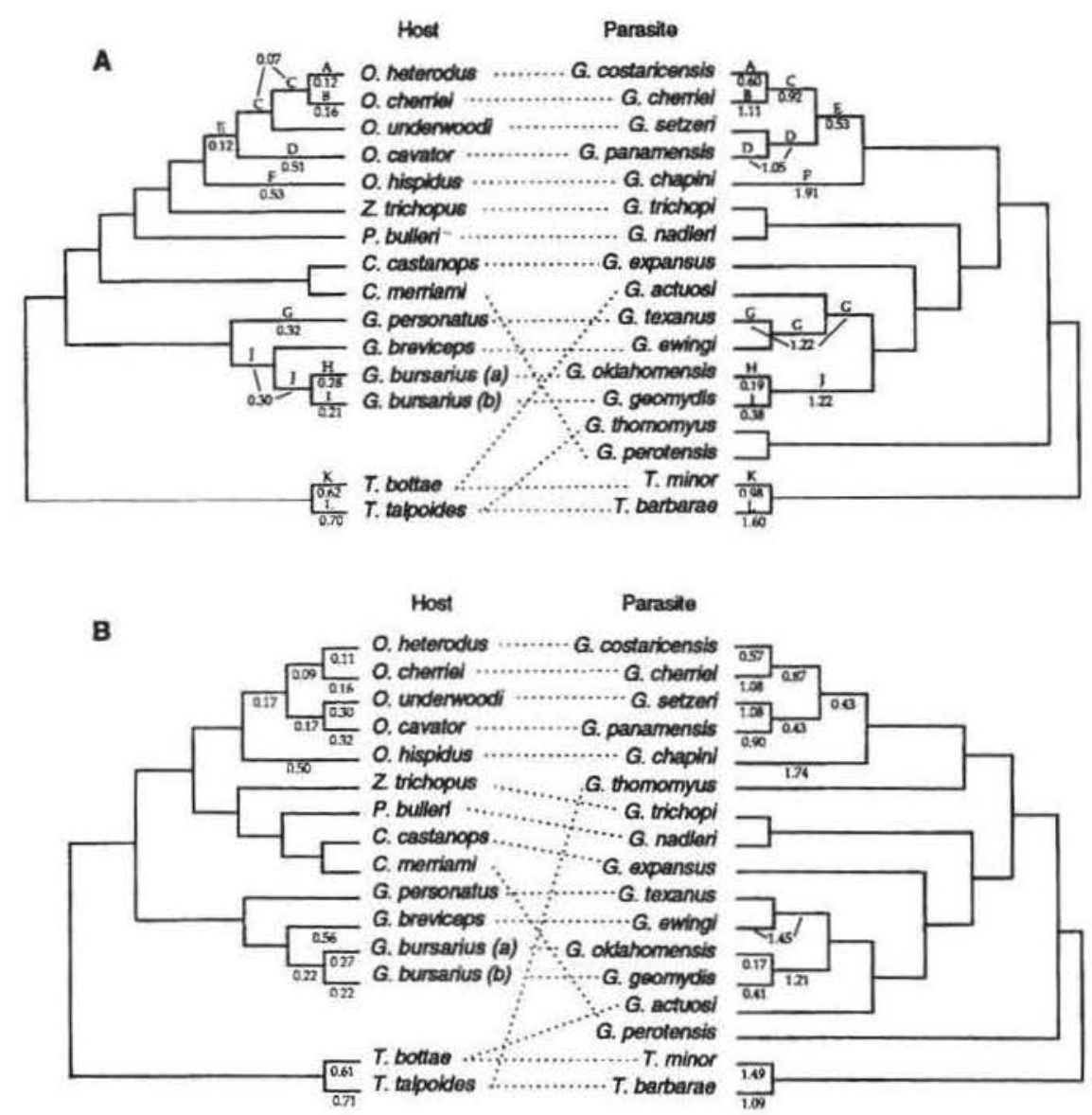

Fig. 2. Phylogenies of pocket gophers (left) and chewing lice (right) generated by analysis of DNA sequences of the gene encoding $\mathrm{COI}$. In each set of trees, coexisting hosts and parasites are linked by dotted lines (each parasite examined was taken directly from the fur of the host individual examined). (A) Host and parasite trees generated by parsimony and maximum-likelihood analyses of the sequence data. (B) Trees generated by Fitch-Margoliash analyses of the host and parasite data. Pocket gopher taxa examined include the genera Orthogeomys, Zygogeomys, Pappogeomys, Cratogeomys, Geomys, and Thomomys. Chewing louse taxa include the genera Geomydoecus and Thomomydoecus. The louse $G$. setzeri also has been reported from $O$. cherriei in areas where the range of $O$. cherriei abuts that of $O$. underwoodi. Relative rates of molecular evolution were investigated by comparison of expected numbers of substitutions per site for hosts and parasites. Maximum-likelihood branch lengths $(x 100)$ based on all nucleotide substitutions are indicated for one of four possible branch combinations in Fig. $2 \mathrm{~A}$ and for one of the two possible combinations in Fig. $28(24)$. Letters above branches indicate branches compared in Fig. 3. Comparisons were restricted to parasites whose phylogenetic history is topologically identical to that of their hosts. Because most of the uncertainty in the phylogenetic analyses involved branches near the base of the trees, only terminal and subterminal branches were compared between gophers and lice. 
analysis (through the origin) and determined that the slopes of the regressions (Fig. 3A) ranged from 2.60 to 2.83 (with a mean of 2.74). This indicates that the overall rate of nucleotide substitution in lice is approximately three times higher than in gophers (27).

To estimate rates of synonymous substitution in gophers and lice, we restricted our analysis of the $\mathrm{COI}$ sequences to fourfold degenerate sites (sites at which all base substitutions are silent). We focused on the largest group of closely related species (Orthogeomys species and their lice) because these species are sufficiently closely related to ensure that corrections for multiple mutations are effective (28). The numbers of variable fourfold degenerate sites in gophers and lice were approximately equal (67 and 69 , respectively). We used a maximum-likelihood model of evolution to infer branch lengths based solely on substitutions at these sites (24). The model included corrections for observed transitional bias (a maximum $4: 1$ bias in gophers; 10:1 bias in lice) and for significantly different nucleotide compositional biases in the two data sets ( $P<$ $0.05)(29)$. These corrections are necessary to estimate evolutionary rates (28), and spurious results should not occur simply because of differences in the number of characters compared or differences in mutational dynamics (and resultant saturation levels) in the two groups being compared. In theory, rates of synonymous substitution are proportional to mutation rates (30), but our estimates cannot be considered direct measures of mutation rates because we have not controlled for possible constraints on the translational apparatus, such as codon bias and second. ary structure of mRNAs.

If nucleotide substitutions at fourfold degenerate sites are selectively neutral,

Fig. 3. Comparison of rates of molecular change in a 379 -bp region of the gene encoding $\mathrm{COI}$ in cospeciated pocket gophers and chewing lice. Letters refer to branches labeled in Fig. 2A. (A) Comparison of maximum-likelihood branch lengths (based on all nucleotide substitutions) for the phylogeny shown in Fig. 2A (one of six possible combinations of cospeciating taxa) (24). Slopes of Model II regressions (through the origin) ranged from 2.60 to 2.83 (with a mean of 2.74 ). which indicates that the overall rate of nucleotide substitution in lice is approximately three times higher than in gophers (27). (B) Comparison of maximum-likelihood branch lengths based solely on nucleotide substitutions at fourfold degenerate sites for Orthogeomys gophers and their lice. Slopes of Model II regressions (through the origin ranged from 9.88 to 11.83 (with a mean of 11.04 ), which indicates that the rate of synonymous substitution in this gene region is approximately an

order of magnitude greater in chewing lice than in pocket gophers. then change at these sites should fit a molecular clock model (30). Accordingly, we tested all possible combinations of cospeciating gophers (Orthogeomys only) and their lice (24) for significant departure from clocklike behavior, using the log-likelihood ratio test (12). In all cases, the data were consistent with molecularclock assumptions, which indicates that substitutions within gophers and within lice accumulate in a roughly time-dependent fashion. Wilcoxon sign-rank tests showed that louse branches were significantly longer than gopher branches in four of the six possible comparisons $(P<$ 0.05 in each case). We used Model II regression analysis (through the origin) to quantify the relation between gopher and louse branch lengths. Slopes of the regressions (Fig. 3B) ranged from 9.88 to 11.83 (with a mean of 11.04), which indicates that the estimated rate of silent substitution for this gene region is approximately an order of magnitude greater in chewing lice than in pocket gophers. Evidence for a higher rate of substitution in lice appears to be independent of the evolutionary model employed, although the magnitude of the rate difference is sensitive to certain parameters of the model (31).

Viewed together, the analysis of all nucleotide substitutions (Fig. $3 \mathrm{~A}$ ) and the analysis of substitutions at fourfold degenerate sites (Fig. $3 \mathrm{~B}$ ) provide insight into the dynamics of molecular evolution for this gene region in the species studied. The analysis of all substitutions indicates that the overall rate of evolutionary change is approximately three times greater in chewing lice than in their hosts (Fig. 3A). Likewise, the means of all pairwise replacement differences for nucleotides and amino acids are approximately three times greater in lice than in gophers (Table 1). In contrast, the analysis of

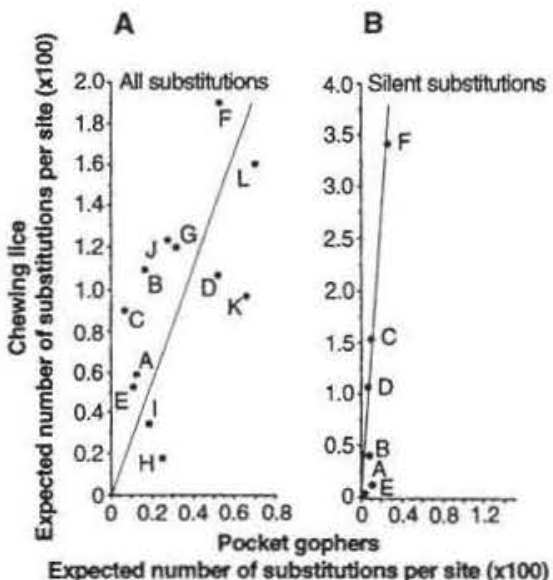

nucleotide substitutions at fourfold degenerate sites indicates that rates of silent substitution in this gene region are roughly 11 times greater in lice than in gophers (Fig. 3B). The fact that this 11 -fold rate difference is not evident when all substitutions are considered is probably the result of selective constraints on replacement substitutions. High levels of functional constraint on the $\mathrm{COl}$ enzyme have been reported in other organisms (28).

The 11-fold difference in rates of synonymous substitution in Orthogeomys gophers and their lice (Fig. 3B) cannot be explained by transition bias or nucleotide frequency bias. Because silent substitutions at the fourfold degenerate sites show clocklike behavior, it is likely that they are neutral or nearly neutral (30). Several possible mechanisms could account for this rate difference, including mutation rate differences caused by possible differences in gene order that affect vulnerability to mutation (32), differences in metabolic rate or general metabolic physiology, generation-time differences, or other factors correlated with body size (33). Alternatively, this rate difference could be caused by mechanisms that are independent of mutation rate, such as codon bias and other constraints on the translational apparatus, or differences in DNA repair efficiency. It is perhaps important that this 11 -fold rate difference is accompanied by a similar difference in generation time between gophers and lice (approximately 1 year in gophers and 40 days in lice) (34). If the observed rate difference results from an underlying difference in mutation rate, then generation time may explain this difference. However, mutation rates are more likely to be influenced directly by nucleotide generation time than by organismal generation time (33). As such, our study suggests that each organismal generation is equivalent to equal numbers of nucleotide generations in pocket gophers and chewing lice. If the 11-fold rate difference reflects a similar difference in mutation rate, then these findings are consistent with the neutral theory of molecular evolution (30), because once the data are corrected for the difference in generation time, they suggest equal rates of mutation per generation in distantly related groups of animals.

\section{REFERENCES AND NOTES}

1. S. A. Nadler, M. S. Hafner, J.C. Hafner, D. J. Hafner. Evolution 44, 942 (1990).

2. R. D. Price and K. C. Emerson, J. Med. Entomol. 8 , 228 (1971).

3. M. S. Hafner and S. A. Nadler, Syst. Zool. 39, 192 (1990): Nature 332, 258 (1988).

4. R. D. M. Page, Syst. Zool. 39, 205 (1990). 
5. J. W. Demastes and M. S. Hafner, J. Mammal. 74. 521 (1993).

6. R. D. M. Page, Int. J. Parasitol. 23, 499 (1993).

7. Voucher specimens are deposited in the Museum of Natural Science, Louisiana State University (LSUMZ) or the New Mexico Museum of Natural History (NMMNH) and are as follows: Orthogeomys under woodi (LSUMZ 29493), O. hispidus (LSUMZ 29231) O. cavator (LSUMZ 29253), O. cherriei (LSUMZ 29539), O. heterodus (LSUMZ 29501), Geomys breviceps (LSUMZ 33940), G. personatus (LSUMZ 31460), G. bursarius halli (LSUMZ 31463; designated " $a$ " in Fig. 2), G. b. majusculus (LSUMZ 31448 designated " $b$ " in Fig. 2), Cratogeomys castanops (LSUMZ 31455), C. merriami (LSUMZ 34343), Pappogeomys bulleri (LSUMZ 34338), Zygogeomys trichopus (LSUMZ 34340), Thomomys bottae (LSUMZ 29320 and 29569), and T. talpoides (NMMNH 1634 and 1637).

8. Pocket gophers were trapped, killed, and immediately brushed to recover lice. Gopher tissues and lice were stored at $-70^{\circ} \mathrm{C}$. DNA extractions from gophers followed the phenol-chloroform tech nique [D. M. Hillis, A. Larson, S. K. Davis, E. A Zimmer, in Molecular Systematics, D. M. Hillis and C. Moritz, Eds. (Sinauer, Sunderland, MA, 1990) pp. 318-370]. DNA extractions from lice followed a modification of the protocol described by $\mathrm{H}$. Liu and A. T. Beckenbach [Mol. Phylogenet. Evol. 1, 41 (1992)] and used two lice from each gopher. One $\mu$ l of the extraction solution was used for DNA amplification in a 50- $\mu$ l reaction. A 379-base pair (bp) region of the mitochondrial COI gene was amplified by polymerase chain reaction (PCR) with two degenerate primers: L6625 (5'-CCGGATC CTTYTGRTTYTTYGGNCAYCC-3') and H7005 (5' -CCGGATCCACNACRTARTANGTRTCRTG$\left.3^{\prime}\right)$. Primer names refer to the $3^{\prime}$ position of each primer relative to the human mitochondrial genome [S. Anderson et al, , Nature 290, 457 (1981)] Double-stranded amplifications were done by four cycles of $1 \mathrm{~min}$ of denaturation $\left(95^{\circ} \mathrm{C}\right), 1 \mathrm{~min}$ of annealing $\left(45^{\circ} \mathrm{C}\right)$, and $1 \mathrm{~min}$ of extension $\left(72^{\circ} \mathrm{C}\right)$ followed by 30 cycles at reduced denaturation temperatures $\left(93^{\circ} \mathrm{C}\right)$ and increased annealing temperatures $\left(60^{\circ} \mathrm{C}\right)$. Methods for production of $\sin$ gle-stranded templates and for sequencing of single-stranded products are given elsewhere (18) Because no two sequences were identical either within or between lice and gophers, cross-contamination is not suspected. Gopher and louse sequences are available through GenBank (accession numbers L.32682 to L32696 for gophers and L32665 to L32681 for lice).

9. D. L. Swofford and G. J. Olsen, in Molecular Systematics, D. M. Hillis and C. Moritz, Eds. (Sinauer, Sun derland, MA, 1990), pp. 411-501; D. M. Hillis, M. W Allard, M. M. Miyamoto, Methods Enzymol. 224, 456 (1993).

10. J.P. Huelsenbeck and D. M. Hilis, Syst. Biol. 42, 247 (1993).

11. We used the PAUP program (Phylogenetic Analysis Using Parsimony, 3.1.1, D. L. Swofford, Illinois Natura History Survey) for phylogenetic analyses of the sequence data. Of the 134 variable sites in pocket go phers (178 in lice), 113 were phylogenetically informative (142 in lice). To compensate for the effects of multiple mutations at nucleotide positions, we used a parsimony analysis in which transitions at first and thirc codon positions were designated as more likely than transversions (with a 10:1 step matrix for gophers and a 17:1 step matrix for lice). These empirical estimates of transition bias represent maximal values, which were observed between taxa with the most similar sequences $(8.7 \%$ sequence divergence between the most similar gophers; $4.7 \%$ between the most similar lice). Because the effects of multiple mutations should be least for closely related taxa, we consider these to be reasonable approximations of the actual transition bias. For each database (gophers and lice) we conducted 100 heuristic searches, varying the addition order of taxa (using the random addition option of PAUP) and using altemate branch-swapping algorithms (using the TBR and SPRoptions). All gopher and louse parsimony trees contained significant phylogenetic signal $\left(g_{1}<-0.54, P<0.01\right)$ [D. M. Hillis and $J$.
P. Huelsenbeck, J. Hered. 83, 189 (1992)]. Gophers in the genus Thomomys and lice of the genus Thomomydoecus were designated as outgroups. The outgroup status of these taxa is supported by previous morphological and molecular analyses $(16,17)$ [R. A. Hellenthal and R. D. Price, J. Kans. Entomol. Soc. 57, 231 (1984); S. A. Nadier and M. S. Hafner, Ann. Entomol. Soc. Am. 82, $109(1989)$.

12. We used PHYLIP IPhylogeny inference Package, $3.5 \mathrm{c}$, J. Felsenstein, Department of Genetics, University of Washington, Seattle] for maximum-likelihood, Fitch-Margoliash W. M. Fitch and E. Margoliash, Science 155, 279 (1967)], and neighborjoining [N. Saitou and M. Nei, Mol. Biol. Evol, 4, 406 (1987)] analyses of the sequence data. Empirical base frequencies and maximal observed transition biases (10:1 for gophers and 17:1 for lice) were used in the maximum-likelihood analysis. The Fitch-Margoliash and neighbor-joining analy. ses were based on distance matrices corrected with the Kimura two-parameter model /substituting maximal observed transition bias values for the default (2:1) value [M. Kimura, J. Mol. Evol. 16. $111(1980)]\}$. We repeated each analysis at least 10 times, varying the input order of taxa using the jumble option of PHYLIP.

13. In the alternative parsimony tree for pocket gophers, the phylogenetic positions of $P$. bulleri and $Z$. trichopus were reversed and the two species of Cratogeomys were not depicted as sister taxa.

14. The neighbor-joining tree differed from the FitchMargoliash tree (Fig. 1B) only in the placement of $P$. bulleri (immediately basal to the five-taxon clade containing $O$. hispidus) and $Z$. trichopus (basal to the eight-taxon clade containing C. castanops, C. merriami, and $P$. bulleri).

15. H. Kishino and M. Hasegawa, J. Mol. Evol. 29, 170 (1989), as used in PHYLIP (12).

16. R. S. Russell, Univ. Kans. Publ. Mus. Nat. Hist. 16, 473 (1968); R. L. Honeycutt and S. L. Williams, J. Mammal. 63, 208 (1982); M. S. Hafner, ibid. 72, 1 (1991).

17. M. S. Hafner, Z. Zool. Syst. Evolutionsforsch, 20, 118 (1982).

18. P. D. Sudman and M. S. Hafner, Mol. Phylogenet. Evol. 1, 17 (1992).

19. In the second parsimony tree, the louse G. actuosi was linked with the oklahomensis-geomydis clade, rather than the texanus-ewingi clade (as in Fig. 2A). The third tree showed $G$. thomomyus near the root of the tree between the outgroups $T$. minor and $T$. barbarae.

20. The neighbor-joining tree was identical to the Fitch-Margoliash tree (Fig. 2B), except that G. thomomyus and $G$. perotensis were linked as sister taxa (as in the parsimony and maximum-likelihood trees, Fig. 2A) and positioned basal to the G. chapini branch,

21. S. A. Nadler and M. S. Hafner, Int. J. Parasitol. 23 , 191 (1993).

22. COMPONENT 2.0, R. D. M. Page, Natural History Museum, London; R. D. M. Page, Syst. Biol. 43, 58 (1994).

23. The test was based on the criterion of minimum number of independent losses necessary to reconcile the host and parasite trees (22). Sixteen of the 20 pairwise comparisons were also found to be statistically significant $(P<0.05)$ according to the criterion of "number of leaves added" (22). Number of leaves added is equivalent to one-half the number of "items of error" [G. Nelson and N. Platnick, Systematics and Biogeography: Cladistics and Vicariance (Columbia Univ. Press, New York, 1981)]. The four comparisons that were not statistically significant (according to the criterion of number of leaves added) all involved the louse parsimony tree that depicted the outgroup Thomomydoecus as polyphyletic. The branching structure of this tree is challenged by evidence trom previous morphological and allozyme studies (11) supporting monophyly of the louse genus Thomomydoecus.

24. The trees (Fig. 2) were reduced to the largest number of taxa that showed identical branching patterns in the hosts and parasites. All possible combinations of cospeciating taxa were compared. For example. there are four possible combinations of nine cospe- ciating taxa in Fig. 2A [that is, the nine-taxon gopher tree can include either $\mathrm{O}$. underwoodi or $\mathrm{O}$. cavator (but not both) and G. breviceps or G. personatus (but not both)]. There are 2 possible combinations of 10 cospeciating taxa in Fig. $2 \mathrm{~B}$ (that is, the tree can include either $G$. breviceps or $G$. personatus, but not both). Because most of the uncertainty in the phylogenetic analyses involved branches near the base of the trees, only terminal and subterminal branches were compared between gophers and lice.

25. N. Mantel, CancerRes, 27, 209 (1967). We calculated distance matrices using the maximum-likelihood model (12). For the 9 cospeciating taxa in Fig. 2A, $t$ $=4.073, P<0.01$. For the 10 cospeciating taxa in Fig. $2 B, t=4.289, P<0.01$. Degrees of freedom were adjusted to $n-1$, where $n=$ number of taxa compared (3).

26. Trees for cospeciating taxa (24) were input as user trees into PHYLIP (12) in order to estimate maximum-likelihood branch lengths. Empirical estimates of transition bias, nucleotide frequency bias, and positional bias (first:second:third codon position: 5:1:40 for gophers and 5:1:21 for lice) were incorporated into the maximum-likelihood model (12). We recognize that the parameters used in maximum-likelihood models will influence rate estimates [K. Fukami-Kobayashi and $Y$ Tateno, J. Mol. Evol. 32, 79 (1991)]. Our models use empirical estimates of these parameters (rather than arbitrary or constant values) to obtain a more realistic approximation of the actual mutational dynamics of the gene region being surveyed $(12,28)$.

27. We used Model II regression analysis (reduced major axis model), which does not permit conventional tests of significance [R. R. Sokal and F. J. Rohlf, Biometry (W. H. Freeman, San Francisco, 1969)]. However, the nonparametric Mantel's test documents a significant relation between genetic distances in gophers and lice (25), and the nonparametric Wilcoxon sign-rank test shows that louse branches are significantly longer than gopher branches. When the regression analyses were not constrained through the origin, none of the $y$-intercepts was significantly different from zero.

28. R. Kondo, S. Horai, Y. Satta, N. Takahata, J. Mol. Evol, 36, 517 (1993); R. A. Capaldi, Annu. Rev. Bio chem. 59, 569 (1990). Total sequence differences among the five species of Orthogeomys range from 4 to $11.9 \%$. Sequence differences among the parasites of Orthogeomys range from 11.9 to $20.3 \%$.

29. Differences in nucleotide composition were tested using the B-statistic (A. P. Martin, G. J.P. Naylor, S P. Palumbi, Nature 357, 153 (1992)].

30. M. Kimura, The Neutral Theory of Molecular Evolution (Cambridge Univ. Press, London, 1983); K. H. Wolfe, P. M. Sharp, W.-H. Li, Nature 337, 283 (1989).

31. The slope of this relationship is affected very little by use of different nucleotide frequency estimates in the maximum-iikelihood model. The slopes are more sensitive, however, to different estimates of transition; transversion bias. Our model used empirical es timates of transitiontransversion bias $14: 1$ in gophers; 10:1 in lice) because such estimates are more likely to reflect the actual mutational dynamics of the gene regions being surveyed $(12,28)$

32. G. G. Brown and M. V. Simpson, Proc. Natl. Acad Sci. U.S.A. 79, 3246 (1982); (28).

33. A.P. Martinand S. R. Palumbi, ibid. 90,4087 (1993): C.-1. Wu and W.-H. Li, bid. 82, 1741 (1985)

34. R. W. Rust, Oecologia 15, 287 (1974); A. G. Marshall, The Ecology of Ectoparasitic Insects (Academ ic Press, London, 1981).

35. We thank D. R. Wolstenholme, R. Okimoto, and C. T Beakley for providing PCR primer sequences and protocols. Photography was provided by R. J. Bou chard. D. H. Clayton, S. V. Edwards, D. A. Good, D. J. Hafner, B. D. Marx, R. D. M. Page, S. Scheiner, and two anonymous reviewers provided helpful advice and comments on this manuscript. Supported by NSF grant BSR-8817329 and NSF-Louisiana Ed ucation Quality Support Fund grant ADP-02. 